\title{
Extracellular-Nitric Oxide-Mediated Platelet-cGMP Production in Type 2 Diabetics Correlates Inversely with Plasma Membrane Cholesterol Levels
}

\author{
Shane Miersch ${ }^{1,4}$, Inga Sliskovic ${ }^{1,3}$, Arianna Vignini $^{2}$, Laura Mazzanti $^{2}$ \\ and Bulent Mutus,
} \author{
Michigan, USA \\ Massachusetts 02141, USA \\ *Corresponding Author: \\ Bulent Mutus \\ Department of Chemistry \& Biochemistry \\ University of Windsor \\ Windsor Ontario, Canada N9B 3P4. \\ Phone: (519) 253-3000 ext. 3533 \\ Fax: (519) 973-7098 \\ E-mail: mutusb@uwindsor.ca
}

${ }^{1}$ Department of Chemistry \& Biochemistry, University of Windsor, Windsor, Ontario, Canada.

${ }^{2}$ Institute of Biochemistry, Università Politecnica delle Marche, Ancona, Italy

${ }^{3}$ Current address: Department of Obstetrics and Gynecology, Wayne State University School of Medicine, Detroit,

${ }^{4}$ Current address: Harvard Institute of Proteomics, Harvard Medical School, 320 Charles Street, Cambridge,

\begin{tabular}{l|l|l|l|} 
Received: 27 April 2009; & Revised: 15 May 2009; & Accepted: 19 May 2009
\end{tabular}

\begin{abstract}
Recently, we showed that the diffusion of nitric oxide (NO) and NO-mediated signaling was affected by the levels of plasma membrane cholesterol in fibroblasts [1]. The generality of these observations, which would imply that perturbations in NO-signaling mediated by increased membrane cholesterol levels, could be a common pathological trigger in vascular cells, was tested in platelets from normal and dyslipidemic type 2 diabetic (T2D) subjects. Plasma LDL-cholesterol correlated directly with platelet plasma membrane cholesterol $\left(\mathrm{Y}=0.28 \mathrm{X}+0.16 ; \mathrm{R}^{2}=0.39\right)$. The average platelet plasma membrane cholesterol concentration was $\sim 2$-fold larger in T2D than in control subjects $(\mathrm{P}<0.05)$. Cyclic GMP production in response to exogenous $\mathrm{NO}$ was $\sim 4$-fold larger in controls than in T2D $(\mathrm{P}<0.05)$. Artificial elevation of membrane cholesterol resulted in $\sim 50 \%$ decrease in the initial rates of NO-uptake. Elevated plasma cholesterol could be a contributing factor to T2D-induced platelet hyperactivity, since it correlated with increased plasma membrane cholesterol, the attenuation of NO-diffusion into platelets and the lowering of cGMP biosynthesis.
\end{abstract}

Keywords: nitric oxide; platelet plasma membrane cholesterol; type 2 diabetes nitric oxide-mediated cyclic GMP production; trans-membrane nitric oxide diffusion. 


\section{Introduction}

Nitric oxide signaling is a complex process uniquely dependent upon its chemical biology and diffusional path from its point of generation to its ultimate targets, both of which limit the spatial distribution of NO to a narrow region. This results in NO having principally a paracrine rather than an endocrine role in the regulation of blood flow and haemostasis.

Although explanations exist for observed hyperaggregability of platelets under hypercholesterolemic conditions [2], a direct role for plasma membrane cholesterol (Chol) as a factor affecting cellular NO-diffusion and signaling has been scantly investigated.

Studies have indicated an inverse relationship between $\mathrm{O}_{2}$ diffusion and plasma membrane Chol content [3]. In a recent publication, we examined the role of the plasma membrane cholesterol in regulating NO diffusion by studying this phenomenon in liposomes, bilayer lipid membranes and fibroblasts defective in cholesterol transport and distribution [1]. These studies clearly indicated that NO-diffusion and signaling was inversely related to the plasma membrane Chol content [1]. A general hypothesis emerging from these studies posits that cholesterol-mediated attenuation of NO-influx and downstream NOsignaling pathways in the vasculature could play a major pathological role in diseases like T2D, often characterized by elevated plasma Chol levels.

Here, blood platelets were employed to test this hypothesis given that they possess negligible ability to biosynthesize cholesterol. Variation in membrane cholesterol content therefore, appears directly related to plasma Chol content [6], perhaps as a result of passive exchange with the pool of non-esterified cholesterol from plasma lipoproteins.

Therefore, the strategy was to compare normal and T2D populations for their plasma lipid profiles, attempt to correlate these with platelet plasma membrane cholesterol levels, and to determine whether the changes in plasma membrane Chol content were reflected in the rates of NO uptake and the extent of cyclic GMP production.

\section{Materials and methods}

Subject selection: Healthy human subjects $(n=10)$, ages 35-70 years were chosen to participate in the study only if they showed no overt symptoms of disease and were taking no medication. Diabetic human subjects, ages 35-70 $(n=10)$ on diet therapy alone and achieving stable and satisfactory glycemic control (fasting glycemia and glycosuria variation $<15 \%$; postprandial glycemia variation $<25 \%$ and $\left.\mathrm{HbA}_{1 c}<7.5 \%\right)$ were chosen for inclusion in the study. None of patients smoked, had history of alcohol abuse or were taking insulin or any drugs known to lower lipids or interfere with the coagulation and antioxidant systems.

The experimental protocols were approved by the Research Ethics Boards, at the University of Windsor and at the Università Politecnica delle Marche.

Materials: 4,5-diaminofluorescein (DAF-2), diethylamine triamine pentaacetic acid (DTPA), methyl- $\beta$-cyclodextrin $(\mathrm{M} \beta \mathrm{CD})$, sucrose, glycerol were obtained from Sigma Chemical (Mississauga, ON). Amplex Red cholesterol oxidase-based cholesterol determination kit was obtained from Invitrogen Canada- (Burlington, ON). cGMP ELISA kits were obtained from Amersham Biosciences - (Piscataway, NJ).

Determination of plasma lipid levels: Plasma lipids (TG, LDL, and HDL) were measured by routine laboratory methods.

Isolation of washed human platelets: Platelets were concentrated into platelet rich plasma (PRP) by centrifugation of whole blood at $190 \mathrm{~g}$ for 30 minutes at $25^{\circ} \mathrm{C}$, then isolated from PRP and washed with 20 volumes of PBS containing 0.5 $\mathrm{mM}$ DTPA. Subsequent experiments were performed in this buffer unless otherwise indicated.

Isolation of platelet plasma membranes: Platelet plasma membranes from both control and diabetic subjects were isolated according to previously published methods [7].

Determination of platelet plasma membrane cholesterol: Gradient-isolated platelet plasma membrane cholesterol was extracted by a 1:1 mix of methanol:chloroform and solvent evaporated under a gentle stream of nitrogen. The resultant 
film was solubilized with $0.1 \mathrm{M}$ phosphate buffer containing $25 \mathrm{mM}$ sodium deoxycholate and $1 \%$ Triton X-100 and cholesterol content quantified according to manufacturers instructions.

Measurement of platelet NO uptake / exclusion: Chol-M $\beta C D$ adduct was prepared according to previously published methods [8] and quantified on the basis of cholesterol content. Plasma membrane cholesterol of $10^{9}$ platelets per $\mathrm{mL}$ was augmented by suspension in a filtered solution of Chol-M $\beta C D$ containing the indicated [cholesterol] for 30 minutes. Following treatment, platelets were centrifuged and resuspended in 100 volumes of buffer. DAF-2 - a cell-impermeant, NO-sensitive fluorophore [9] was added to the platelet suspension to a final concentration of 10 $\mu \mathrm{M}$. Platelets were then treated (in the presence of DAF-2) with $1 \mu \mathrm{M}$ DEA-NO [10] in a microwell plate at a count that consumed approximately $50 \%$ of delivered NO. Formation of nitrosated DAF (DAF-2T) was monitored as evolution of fluorescence on a Perkin-Elmer Victor 3 using an excitation emission couple of 438 / $535 \mathrm{~nm}$.

Platelet cGMP measurements: A standard curve for cGMP detection was prepared as per manufacturer's instruction. Briefly, varying cGMP concentrations $(2-512 \mathrm{fmol})$ were placed in the 96-well plate and incubated with reaction mixture containing cGMP conjugated to horseradish peroxidase. Addition of TMB substrate resulted in a color development which can be monitored by measuring absorbance at 630 nm. Similarly, platelet samples $\left(10^{8}\right)$ previously exposed to L-NMMA, an inhibitor of endogenous $\mathrm{NO}$ generation, were exposed to the $\mathrm{NO}$ donor SNAP $(100 \mu \mathrm{M})$, for 30 minutes at $37^{\circ} \mathrm{C}$ followed by cGMP measurements interpolated from the standards.

\section{Results}

Platelet membrane Chol correlates with LDLChol: Control $(\mathrm{n}=10)$ and T2D subjects $(\mathrm{n}=10)$ were probed for their plasma TG, LDL-, HDLChol as well as total Chol. Platelet plasma membrane samples from these subjects were isolated by sucrose gradient centrifugation and cholesterol quantified by enzymatic assay. There was no correlation between TG, total-Chol and HDL-Chol with platelet plasma membrane, however, plasma membrane Chol could be correlated with LDL-Chol $\quad(\mathrm{Y}=0.28 \mathrm{X}+0.16$; $\mathrm{R}^{2}=0.39$; Fig 1).

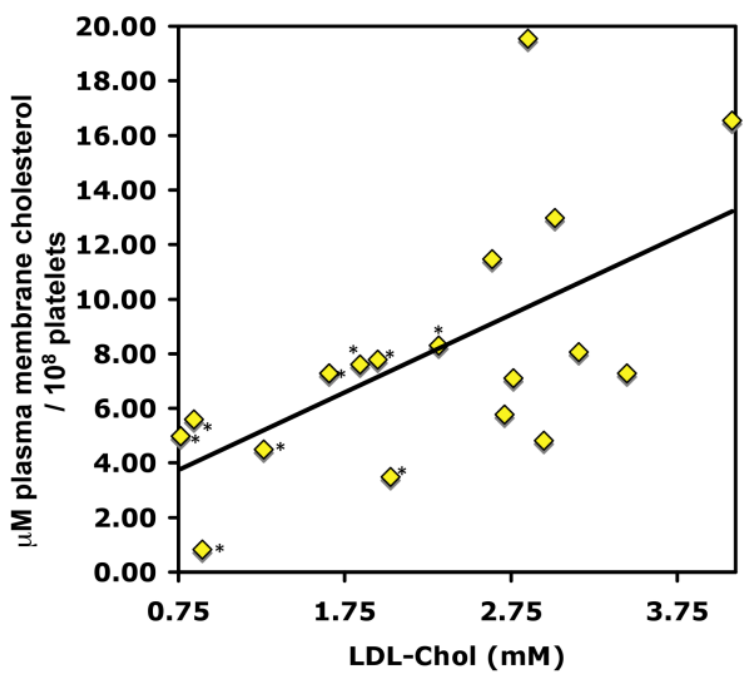

Figure 1- Plot of plasma [LDL-Chol] vs. [platelet plasma membrane cholesterol] from normal (*) and T2D subjects. The best fit of the data yielded a positive correlation with the equation $\mathrm{Y}=0.28 \mathrm{X}+0.16$; $\mathrm{R}^{2}=0.39$ ) between the two, thus suggesting that platelets membrane cholesterol content varies with the prevailing plasma cholesterol levels.

These results are in agreement with previous studies, suggesting that the plasma membrane Chol content of blood cells like platelets [6] and erythrocytes [11], (lacking intracellular cholesterol trafficking and biosynthetic pathways) varies directly with the plasma lipoprotein Chol pool.

NO-diffusion into platelets is inhibited upon elevation of platelet plasma membrane Chol: The use of Chol-loaded M $\beta C D$ to augment the Chol content of plasma membranes is a well established technique [8]. Here we obtained platelets from normal subjects and elevated their plasma membrane Chol by incubation with Chol-loaded $\mathrm{M} \beta C D$. We then compared NO uptake amongst control and Chol-loaded platelets. In these experiments, NO was delivered from a common NO donor to a suspension of platelets in a solution containing DAF-2; a cell-impermeant fluorophore reactive to $\mathrm{NO}$ oxidation products $\left(\mathrm{NO}_{\mathrm{x}}\right)$. With this experimental design, a fraction of NO will 
diffuse into platelets and be unavailable for reaction with DAF, thus avoiding interferences arising from the intracellular environment. If plasma membrane cholesterol contributes to a barrier to NO-diffusion, logic suggests that as membrane cholesterol content increases, more NO will remain in the extracellular environment, thus enhancing its reaction with DAF-2 observed as an increase in fluorescent $\mathrm{NO}_{\mathrm{x}}$-reaction product per unit time. This is precisely what was observed in these experiments. The initial rates of the fluorescent DAF-nitrosation was increased by 1.6-fold in Chol-loaded platelets (Fig. 2, open diamonds) in comparison to control platelets (Fig. 2, yellow triangles). We have determined previously that $40 \mu \mathrm{M}$ M $\beta C D$-Chol treatment increased PM-Chol by $\sim 1.3$-fold (data not shown). Figure 3 reveals a $\sim 2$ fold increase in membrane cholesterol of platelets from Type 2 diabetics and would likely translate into increased extracellular DAF nitrosation. Although no direct comparison between extracellular DAF nitrosation and intracellular cGMP generation has been attempted, the observation of $\sim 4$-fold lowering of cGMP production in platelets from T2D patients versus controls suggests that there may be components other than that mediated by increased PMmembrane Chol that attenuate platelet cGMP production in diabetes. Regardless, changes in DAF nitrosation observed following Chol- loading experiments strongly suggest that NO-diffusion into platelets is impeded by elevations in plasma membrane Chol.

Increased platelet plasma cholesterol translates into attenuated NO-signaling: In order to determine whether platelets with naturally varying plasma Chol levels also exhibited differences in response to NO, we assessed the formation of cGMP in response to exogenously applied NO and sought correlation with membrane cholesterol. We first exposed an intact aliquot of the platelet samples utilized in membrane Chol determinations, to L-NMMA, a nitric oxide synthase inhibitor, for $5 \mathrm{~h}$ to block all intraplatelet NO biosynthesis. These platelets were then exposed to $100 \mu \mathrm{M}$ SNAP and their intracellular cGMP production was determined with 30 min of NO exposure. Platelets from control subjects had 2-fold lower plasma membrane Chol than T2D (Fig 3, yellow squares). The NO-induced, cGMP production in these two populations were inversely proportional to their plasma membrane Chol-levels, where control platelets exhibited $~ 4-$ fold larger cGMP production in comparison to T2D treating with equal [NO] (Fig 3, blue squares). It is worth noting that basal levels of cGMP were elevated in diabetic patients versus healthy controls, however stimulation with NO abolished this difference. Increased blood glucose is thought to activate the cGMP pathway by an osmotic mechanism that involves PKC- $\beta$ [12] and explains the disparity in basal cyclic nucleotide levels. Despite this disparity, the response to NO in platelets from diabetics appears blunted and correlates inversely with platelet cholesterol levels.

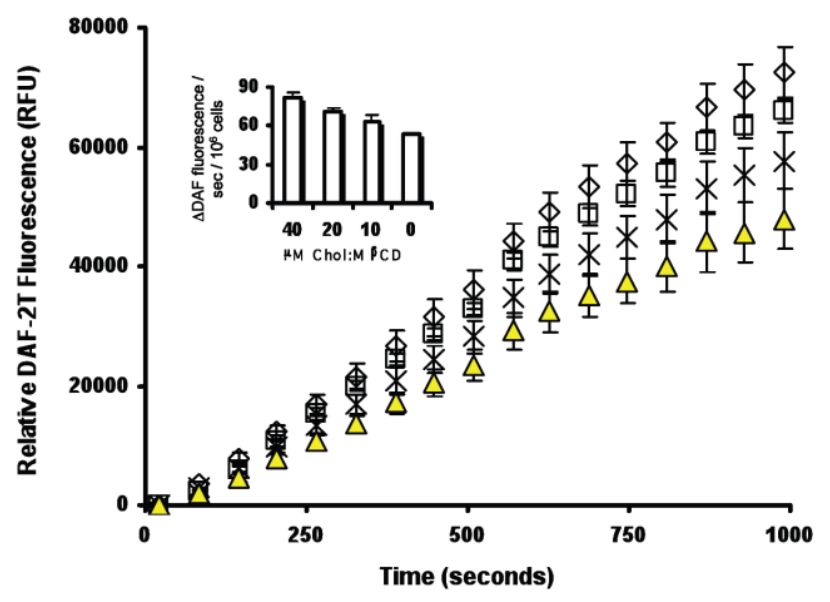

Figure 2- Platelets were treated with 0 (yellow diamonds), 10 (X) , 20 (unfilled squares) and $40 \mu \mathrm{M}$ (unfilled diamonds) Chol:M $\beta C D$ for 15 minutes to augment membrane cholesterol, isolated by centrifugation and resuspended in buffer containing 10 $\mu \mathrm{M}$ DAF-2. DEANO $(1 \mu \mathrm{M})$ was added to five replicate samples in a microwell plate and the evolution of fluorescence monitored at $595 \mathrm{~nm}$ (excitation $545 \mathrm{~nm}$ ). Results are representative of three consecutive experiments, error bars indicating standard deviation and the inset shows comparison of the effect of cholesterol-addition to platelet plasma membrane on the initial rates of DAF-2T formation. 


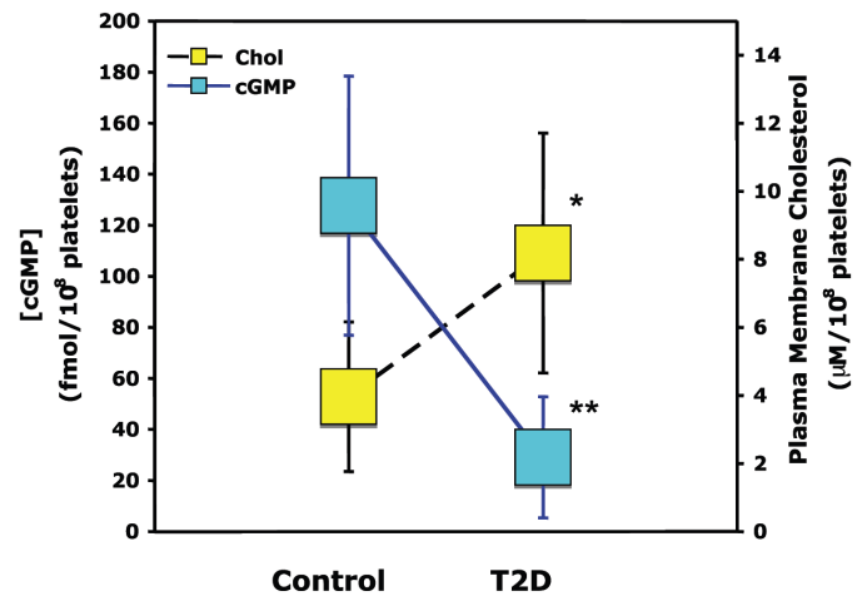

Figure 3-Plots of average [platelet plasma Chol] (yellow squares) and intraplatelet cGMP production (blue squares) from control and T2D subjects. Error bars indicate standard deviation $(n=10)$, the significance of the data was determined with the t-test (2-tailed, 2 sample equal variance) $* \mathrm{P}<0.05$, ** $\mathrm{P}$ $<0.05$

\section{Discussions}

The aim of this study was to test whether the platelet plasma membrane Chol-content affected NO-diffusion and downstream signaling i.e. cGMP production. Loading of platelet membranes with cholesterol clearly impeded the entry of NO into cells, leaving more NO to react with DAF-2, thus resulting in $\sim 1.5$-fold larger initial rates of $\mathrm{NO}_{\mathrm{x}}$-mediated $\mathrm{DAF}-2 \mathrm{~T}$ formation in comparison to control cells.

We then investigated the relationship between platelets bearing natural variations in their plasma membrane Chol levels and the amount of cGMP production in response to extracellular generation of NO. Prior to conducting these experiments we first assessed whether variations in human plasma lipid profiles would correlate with the Chol content of their platelet membranes. This turned out to be the case as a direct correlation between the LDL-Chol levels of normal and T2D subjects and their plasma membrane Chol-levels was obtained confirming previous studies in both platelets [6] and erythrocytes [3]. Cellular cholesterol levels are generally regulated by a balance of endogenous synthesis in the ER, receptor-mediated endocytosis of lipoproteins and efflux to lipid-deficient HDL particles. Although platelets are reported to possess a functional ABCA1 receptor [13] (and may thus be able to efflux cholesterol to HDL), the absence of LDL-mediated receptor regulation has been observed [14] perhaps explaining the correlation with increasing LDL, but not HDL. The absence of correlation between platelet cholesterol with HDL has been noted by other groups [15] and in general cholesterol efflux from platelets remains poorly characterized.

Analysis of NO-induced cGMP responses in platelets from T2D patients bearing increased membrane cholesterol versus normal platelets dramatically revealed that as the average platelet plasma membrane Chol levels approximately doubled the cGMP production decreased by $\sim 4-$ fold. This is very significant since the platelet inhibitory effects of $\mathrm{NO}$ on platelet aggregation are mediated by cGMP [16].

The data presented here strongly support the hypothesis that NO-uptake and ultimately NOsignaling can be affected by elevations in plasma membrane cholesterol. The present observations with platelets are in agreement with our recent study where sterol transport-defective NiemannPick type C1 (NPC1) fibroblasts exhibited increased plasma membrane cholesterol content but decreased activation of both intracellular soluble guanylyl cyclase and vasodilatorstimulated phosphoprotein (VASP) phosphorylation at $\operatorname{Ser}(239)$ induced by exogenous NO exposure relative to their normal human fibroblast (NHF) counterparts. Clearly, these effects were related to plasma membrane Chol levels since its augmentation, in NHF diminished production of both cGMP and VASP phosphorylation elicited by NO to NPC1comparable levels [1].

In addition to its cholesterol lowering properties, statins are also known to provide cardiovascular benefits. Our group and others have observed mitigation of diabetes-associated hyperaggregability upon administration of statins [17]. This effect has generally been attributed to the pleiotropic effects of statins mediated in part by inhibition of isoprenoid formation required for 
the functional assembly of NADPH oxidase and concomitant anti-oxidant effects.

The work outlined in this current paper would suggest that the anti-platelet and vasculoprotective effects of statins could also stem directly from their cholesterol-lowering properties observed as a decrease in platelet membrane cholesterol content and concomitant increase in NO anti-aggregatory bioactivity.

Conclusions: The significance of the present study is that it demonstrates the entry of NO, and the biosynthesis of its second messenger, cGMP, a well established platelet inhibitor, can be inhibited by elevations in plasma membrane Chol in response to T2D-dependent hyperlipidemia. The plasma membrane Chol-mediated attenuation of intracellular NO-signaling could therefore be a pivotal control point in tipping the balance in the T2D-platelet to the pro-aggregatory side.

Abbreviations: 4,5-diaminofluorescein, DAF-2; methyl- $\beta$-cyclodextrin $\mathrm{M} \beta \mathrm{CD}$; nitric oxide, NO; type 2 diabetes mellitus, T2D.

\section{Acknowledgements}

This work was supported by a Discovery Grant from the Natural Sciences and Engineering Research Council (NSERC) to BM.

\section{References}

1. Miersch S.; Espey, M.G.; Chaube, R.; Akarca, A.; Tweeten, R.; Ananvoranich, S.; Mutus, B. Plasma membrane cholesterol content affects nitric oxide diffusion dynamics and signaling. J Biol Chem. 2008, 283, 18513-18521.

2. Schmitz, G.; Schambeck, C.M. Molecular defects in the ABCA1 pathway affect platelet function. Pathophysiol Haemost Thromb, 2006, 35, 166-174.

3. Buchwald, H.; Menchaca, H.J. Michalek V.N.; Rohde T.D.; Hunninghake D.B.; O'Dea T.J. Plasma cholesterol: an influencing factor in red blood cell oxygen release and cellular oxygen availability. J Am Coll Surg 2000, 191, 490-497.

4. Porterfield, D.M.; Laskin, J.D.; Jung,; Malchow, R.P.; Billack, B.; Smith, P.J.; Heck,
D.E. Proteins and lipids define the diffusional field of nitric oxide. Am J Physiol Lung Cell Mol Physiol, 2001, 281, L904-912.

5. Chiu, S.W.; Jakobsson, E.; Mashl, R.J.; Scott, H.L. Cholesterol-induced modifications in lipid bilayers: a simulation study. Biophys $J$, 2002, 83, 1842-1853.

6. Le Quan Sang, K.H.; Mazeaud, M.; Astarie, C.; Duranthon, V.; Driss, F.; Devynck, M.A. Plasma lipids and platelet membrane fluidity in essential hypertension. Thromb Haemost, 1993, 69, 70-76.

7. Barber, A.J.; Jamieson, G.A. Isolation and characterization of plasma membranes from human blood platelets. J Biol Chem, 1970, 245, 6357-6365.

8. Christian, A.E.; Haynes, M.P.; Phillips, M.C.; Rothblat, G.H. Use of cyclodextrins for manipulating cellular cholesterol content. $J$ Lipid Res 1997, 38, 2264-2272.

9. Kojima, H.; Nakatsubo, N.; Kikuchi, K.; Kawahara, S.; Kirino, Y.; Nagoshi, H.; Hirata, Y.; Nagano, T. Detection and imaging of nitric oxide with novel fluorescent indicators: diaminofluoresceins. Anal Chem 1998, 70, 2446-2453.

10. Davies, K.M.; Wink, D.A.; Saavedra, J.E.; Keefer, L.K. Chemistry of the diazeniumdiolates. 2. Kinetics and mechanism of dissociation to nitric oxide in aqueous solution. J Am Chem Soc 2001, 123, 54735481.

11. Cazzola, R.; Rondanelli, M.; Russo-Volpe, S.; Ferrari, E.; Cestaro, B. Decreased membrane fluidity and altered susceptibility to peroxidation and lipid composition in overweight and obese female erythrocytes. $J$ Lipid Res 2004, 45,1846-1851.

12. Massucco, P.; Mattiello, L.; Russ, I.; Traversa, M,; Doronzo, G.; Anfossi, G.; Trovati, M. High glucose rapidly activates the nitric oxide/cyclic nucleotide pathway in human platelets via an osmotic mechanism. Thromb Haemost. 2005, 93(3):517-26.

13. Nofer, JR.; Herminghaus, G.; Brodde, M.; Morgenstern, E.; Rust, S.; Engel, T.; Seedorf, U.; Assmann, G.; Bluethmann, H.; Kehrel, BE. Impaired platelet activation in familial high 
density lipoprotein deficiency (Tangier disease). J Biol Chem. 2004, 279(32):34032-7.

14. Ravindran, R.; Krishnan, L.K. Increased platelet cholesterol and decreased percentage volume of platelets as a secondary risk factor for coronary artery disease. Pathophysiol Haemost Thromb. 2007, 36(1), 45-51.

15. García-Guerra, R.; Mateo, C.R.; GarcíaMuñoz, M.S.; Zapata, M.A.; Plaza, I,; González-Rodríguez, J. Distinct cholesterol and phospholipid incorporation at the platelet plasma membrane of hyperlipidemic subjects: structural order and function. Platelets. 1998, 9(6), 365-73.

16. Sala, C.; Rescaldani, M.; Santin, E.; Bolla, G.; Cuspidi, C. Clinical and Functional Correlates of Platelet Cyclic GMP in Essential Hypertensives. Am J Hypertens. 2009 Mar 26. [Epub ahead of print].

17. Miersch, S.; Sliskovic, I.; Raturi, A.; Mutus, B. Antioxidant and antiplatelet effects of rosuvastatin in a hamster model of prediabetes. Free Radic Biol Med. 2007, 42(2), 270-9. 